Delft University of Technology

\title{
Design and Validation of a Passive Camber Morphing Concept for Rotorcraft
}

Zahoor, Y.; Sodja, J.; De Breuker, R.

DOI

10.2514/6.2022-0173

Publication date

2022

Document Version

Final published version

Published in

AIAA SCITECH 2022 Forum

\section{Citation (APA)}

Zahoor, Y., Sodja, J., \& De Breuker, R. (2022). Design and Validation of a Passive Camber Morphing Concept for Rotorcraft. In AIAA SCITECH 2022 Forum [AIAA 2022-0173] (AIAA Science and Technology Forum and Exposition, AIAA SciTech Forum 2022). https://doi.org/10.2514/6.2022-0173

\section{Important note}

To cite this publication, please use the final published version (if applicable).

Please check the document version above.

\section{Copyright}

Other than for strictly personal use, it is not permitted to download, forward or distribute the text or part of it, without the consent of the author(s) and/or copyright holder(s), unless the work is under an open content license such as Creative Commons.

\section{Takedown policy}

Please contact us and provide details if you believe this document breaches copyrights.

We will remove access to the work immediately and investigate your claim. 


\title{
Design and Validation of a Passive Camber Morphing Concept for Rotorcraft
}

\author{
Yasir Zahoor, * Jurij Sodja ${ }^{\dagger}$ and Roeland De Breuker. ${ }^{\ddagger}$ \\ Delft University of Technology, 2629 HS, The Netherlands \\ Mark Voskuij ${ }^{\S}$ \\ Netherlands Defence Academy, 1780 AC, The Netherlands
}

This paper presents an overview of the design and validation of a novel passive camber morphing system for rotorcraft. The passive system works for a variable speed rotor where a potential increase in pilot control authority and power reduction is possible. In the proposed concept, the rotor speed is varied by $10 \%$ to change the apparent centrifugal force which is used to morph a trailing edge flap by a mechanical system. In this context, the important design parameters in relation to the passive morphing concept are introduced, after which the working principle of the concept is explained. The design and development of the test demonstrator, experiments in a whirl tower setup, and test findings are also presented. The results indicate that a passive trailing edge morphing concept is feasible and has the potential to be used in a variable speed rotor to achieve the desired performance benefits.

\section{Nomenclature}

$\beta=$ flap deflection, rad

$\varphi=$ angle of the inclined surface of the spanwise shaft, $\mathrm{rad}$

$\theta_{o}=$ blade collective pitch, rad

$\omega=$ rotor speed, $\mathrm{rad} / \mathrm{sec}$

$C_{P}=$ rotor power coefficient

$C_{T}=$ rotor thrust coefficient

$F_{c}=$ centrifugal force, $\mathrm{N}$

$F_{S}=$ spring restoring force, $\mathrm{N}$

$k_{s} \quad=$ spring stiffness, $\mathrm{N} / \mathrm{mm}$

\footnotetext{
*PhD Researcher, Aerospace Structures and Computational Mechanics, Faculty of Aerospace Engineering, Kluyverweg 1; Delft, The Netherlands. ${ }^{\dagger}$ Senior Researcher, Aerospace Structures and Computational Mechanics, Faculty of Aerospace Engineering, Kluyverweg 1; Delft, The Netherlands

$¥$ Associate Professor, Aerospace Structures and Computational Mechanics, Faculty of Aerospace Engineering, Kluyverweg 1; Delft, The Netherlands, AIAA Senior member.

${ }^{\S}$ Professor, Faculty of Military Sciences; Den Helder, The Netherlands, AIAA Member.
} 


$$
\begin{aligned}
& p_{L}=\text { spring preload, } \mathrm{N} \\
& x_{s}=\text { spanwise stroke, } \mathrm{mm} \\
& x_{t}=\text { chordwise stroke, } \mathrm{mm}
\end{aligned}
$$

\section{Introduction}

Morphing is a capability that introduces smooth shape changes of a wing and control surfaces with respect to a particular flight condition. This results in a potential increase of in-flight performance of both fixed-wing aircraft and rotorcraft [1]. In an aircraft, morphing can be achieved by utilizing both active and passive means. An active actuation system works by using specific actuators for controlling the behaviour of the morphing control surface [2, 3]. In such cases, both the input and output are continuously monitored and controlled through a feedback system. A passive system, on the other hand, requires no extra onboard source or energy for actuation and relies solely on either the aerodynamic loads, temperature, or centrifugal loads (in rotorcraft) to change the shape of the surface while maintaining sufficient stiffness. The advantage of such a passive system lies in the fact that the required shape changes are introduced without an additional control system. In addition, passive morphing is easier to implement since it does not require additional feedback-driven actuation mechanisms, whereas active morphing may require sophisticated onboard signal processing, large dimensional controllers, rapid continuous actuation mechanisms, as well as computational power [4].

The idea of passive morphing in rotorcraft arises from the need to utilize the tremendous amount of centrifugal force generated in a rotorcraft, which can range from a force of $60,000 \mathrm{~N}$ to $120,000 \mathrm{~N}$ at the blade root of two to four passenger helicopters and up to 40 tons for larger helicopters [5]. However, this centrifugal force needs a mechanism so that it can be utilized in a controlled manner for passive actuation. Since the magnitude of the centrifugal force is proportional to the square of the speed, controlling the rotor speed (RPM) can effectively change the centrifugal force. This makes centrifugal force operated passive morphing systems suitable for a variable speed rotor (VSR) which allows variation in rotor RPM in a controlled manner.

The studies of VSR indicate that varying rotor speed is advantageous for flight performance, despite the additional complexities as compared to a fixed-RPM rotorcraft. For instance, in a study performed by Han et al., it is shown that for a flight at $70 \mathrm{~m} / \mathrm{s}$, when the rotor rotational speed is decreased to $82 \%$ of the nominal speed, power is reduced by $40 \%$ for the same output thrust that is required at constant rotor speed [6]. While using a VSR system, it is also important to realize that multiple aspects such as flight envelope extension, power optimization, and fuel consumption benefit from its use. Hence, the technology is promising, and maybe an indispensable feature for future rotorcraft configurations [7]. For instance, in terms of fuel efficiency, researchers have reported fuel reductions in the range of about 10-13\% for a VSR as compared to standard constant-speed rotor operation [8, 9]. In addition, generalized treatments of helicopter rotor theory have long indicated that, for a given disk loading and solidity, the choice of rotor-blade tip speed greatly affects the 
performance of helicopters in the hovering and forward flight conditions and that the tip-speed requirements in the two flight conditions are conflicting [10]. This indicates another potential source of performance benefit. As true airspeed increases, advancing tip Mach numbers become large, and retreating blade stall occurs, leading to increased vibration. This behaviour can be alleviated by slowing the rotor speed [11]. There are quite a few examples of helicopters that have been developed with variable-speed capability, the earliest one being MacDonnel-XV1. One of the features of the $\mathrm{XV}-1$ was that in airplane mode, the rotor would be slowed to a significantly lower speed to reduce its drag in forward flight [12]. Other notable examples include Bell's Eagle Eye, Boeing's A-160 Humming Bird, and Airbus Helicopter's EC-145.

In terms of speed ranges for VSR, researchers have explored different ranges in the past. Han et al. [13] mentions rotor speed change in the range of $(100 \%-53.8 \%),(100 \%-31.5 \%)$ and $(100 \%-39.2 \%)$, explaining that increasing the range of the variation of the rotor speed is beneficial to the helicopter performance. Also, the influence of speed reduction in hover, low-speed, and high speed can be different [14]. In the studies performed by Han et al. [15], the helicopter performance benefits by VSR and variable blade twist are investigated. It is found that the combination of VSR and variable blade twist can obtain more power reduction than individually varying rotor speed or blade twist. This is an important outcome and encourages further studies to observe the performance of camber morphing coupled with the VSR.

Due to the potential advantages of the VSR discussed in the preceding paragraphs and the need for passive actuation using centrifugal force, studies have been performed in the past to develop different morphing concepts. Most of the work in this domain has been carried out for span and chord morphing. In these concepts, morphing is achieved by variations in the apparent centrifugal force due to the RPM change. For instance, a chord morphing system based on the cellular structure is designed and tested [16] that shows its significance for stall alleviation [17]. Similarly, another concept for chord morphing utilizes centrifugally operated Von Mises Truss to achieve the desired objectives of main rotor power reductions of nearly $17 \%$ [18]. In a similar work for span morphing, a proof-of-concept demonstration rotor is designed, fabricated, and tested on a hover stand [19]. This concept relies on a spring-based mechanism that works through the changes in the RPM and shows power reductions of around 10\% when compared to its baseline configuration. However, the author has been unable to find any study that relates the variation in RPM to camber morphing, despite being one of the most popular choices for active morphing in rotorcraft.

In view of the aforementioned discussion, the current paper presents the design, development, and testing of a demonstrator that changes the shape of the trailing edge (TE) flap by variations in rotor RPM in a specific range. The discussion in this paper is organized as follows. Section II describes the significance of passive camber morphing with respect to VSR. Section III provides an overview of the passive morphing concept and the important parameters for its design. Section IV discusses the design of the demonstrator, which is built for the proof-of-concept and also presents the details of the overall test setup that serves to validate the operation of the passive system. Section $\mathrm{V}$ provides a 
discussion on the test results and the operation of the passive system. In the end, Sec. VI mentions conclusions related to the design work and test campaign.

\section{Significance of Passive Morphing with VSR}

Before discussing the passive morphing system in detail, it is important to establish its importance in relation to VSR. For this purpose, Translation Induced Camber (TRIC) morphing concept, which was envisaged for a fixed-wing aircraft is used [20]. In this context, it is worth mentioning that the author has recently implemented the TRIC concept to deflect a trailing edge flap of a rotor blade by means of piezoelectric actuators [21]. Recent work by Vidyarthi et al. [22] has indicated that if the same morphing concept is coupled passively to a VSR, power benefits and an increase in control authority of the pilot can be achieved. For this purpose, a numerical tool called Helicopter Conceptual Design and Performance Analysis Tool (HOPLITE) developed by Vidyarthi et al. [23] is utilized, which is based on the Blade Element Theory to model the main rotor and accounts for changes in the rotor blade geometry, including the camber morphing. The induced velocity over the disk is calculated using Glauert's formulations [24]. This tool combines various low-fidelity methods, which are described by Vidyarthi et al. [23], such that quick design assessments can be performed for aerodynamic efficiency, power consumption, and engine emissions. On the basis of the aforementioned analysis tool, a detailed numerical analysis for both the active and passive morphing concepts is presented by Vidyarthi et al. [22]. However, a brief account of the key performance benefits is highlighted in this section to indicate the importance of the passive camber morphing concept. As mentioned in Sec. I. the passive morphing concepts for rotorcraft are driven by the apparent centrifugal forces experienced by a rotor. The variation in rotor RPM can be used to change the centrifugal forces so that the morphing can be achieved in a controlled manner. Considering the Bo105 rotor as the baseline rotor, two flight conditions are studied, namely, hover at sea level and hover at an altitude of $2,000 \mathrm{~m}$. In terms of performance parameters, collective pitch normalized with rotor thrust coefficient $\theta_{0} / C_{T}$ and rotor power coefficient normalized with thrust coefficient $C_{P} / C_{T}$ are used [22]. In the study, the flap is placed between $70 \%$ and $90 \%$ of the rotor radius. This is because the dynamic pressure around this location on the rotor blade keeps the morphing flap effectiveness at a high level resulting in a substantial impact on the local aerodynamics of the rotor when the flap is actuated. The rotor RPM is changed from $100 \%$ to $90 \%$, and the flap deflections are varied between $0^{\circ}$ and $6^{\circ}$ for each case. The weight of the aircraft is taken as $2200 \mathrm{~kg}$, approximately $90 \%$ of the maximum take-off weight [25]. The outcome of the performance analysis is presented in Fig. 1.

Figure $1 \mathrm{a}$ and $1 \mathrm{~b}$ show respectively the power and collective pitch requirements to trim the rotor at sea-level. It can be seen that power reduction is mainly linked to the rotor RPM and are according to the studies found in literature [15, 26]. It is also observed that slowing the rotor has more influence than camber morphing. Nevertheless, the reduction in collective is around $10 \%$ which is quite significant and is an indirect measurement of pilot control authority [22]. For the edge of the flight envelope case, such as hover at $2000 \mathrm{~m}$, rotor morphing has shown to be more useful [27]. 


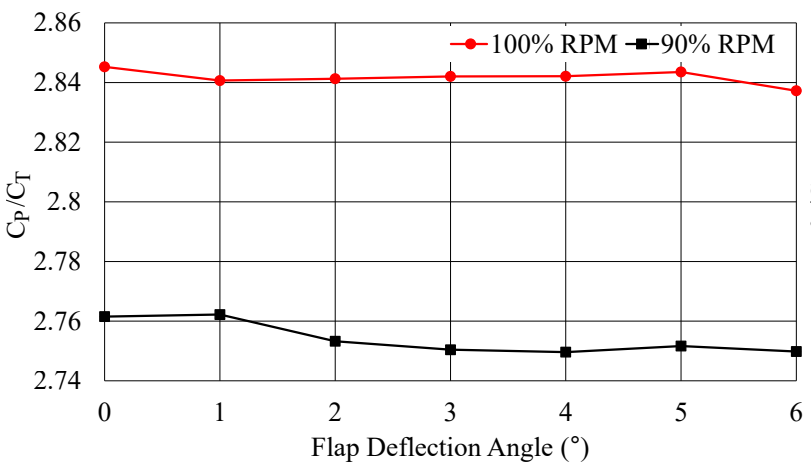

(a) Rotor power reduction at sea level.

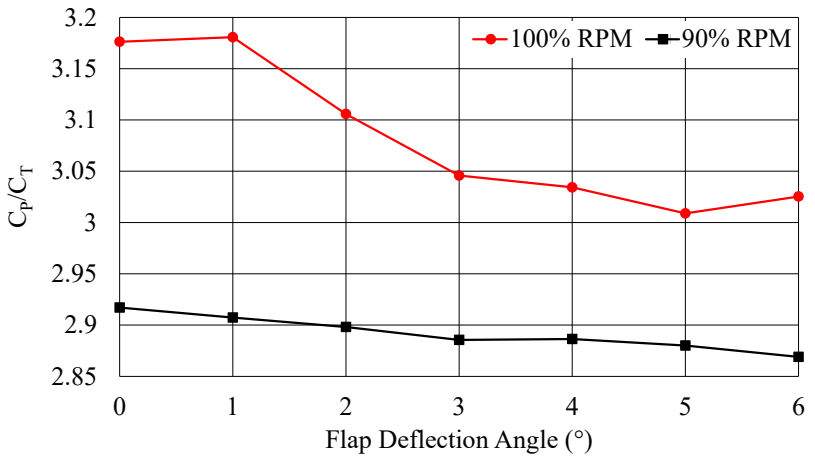

(c) Rotor power reduction at $2000 \mathrm{~m}$.

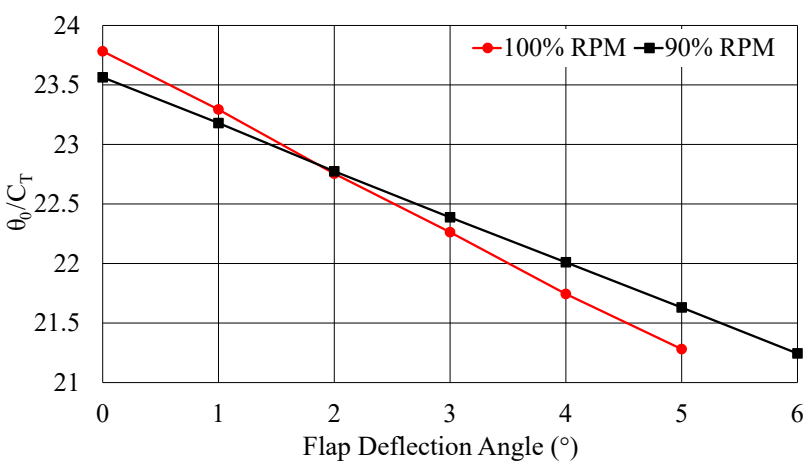

(b) Collective pitch reduction at sea level.

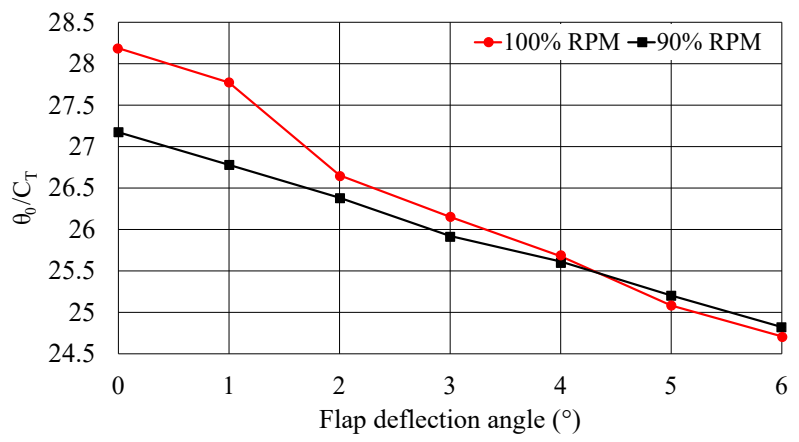

(d) Collective pitch reduction at $2000 \mathrm{~m}$.

Fig. 1 Power and collective pitch reductions due to passive morphing.

Figures $1 \mathrm{c}$ and $1 \mathrm{~d}$ show a greater performance benefit when the RPM reduction is coupled with passive morphing. A $12 \%$ and $8 \%$ reduction in collective pitch and power required to trim the rotor are observed, respectively.

It is clear from the results of the aforementioned study that passive morphing has potential benefits when coupled with a VSR, especially when the operational requirements are hover intensive. A passive morphing flap reduces the collective pitch requirement in a variable rotor speed rotorcraft, which increases the pilot control authority and can potentially extend the flight envelope. More details on the performance benefits associated with the passive morphing concept are provided in Ref. [22].

\section{Overview of the Passive Camber Morphing Design}

The passive camber morphing concept is presented in this section. It highlights the important requirements and parameters in the design process.

\section{A. Proposed Concept for Passive Morphing}

As mentioned in Sec. III the TRIC morphing concept presented in Fig. 2 is used for passive morphing in VSR. The top skin of the flap is fixed to the rotor blade structure. The bottom skin, which translates in the chordwise direction, is constrained in the out-of-plane direction. A centrifugal-force-operated mechanism is placed inside the blade structure, 
causing the bottom skin to translate. The resulting translation of the bottom skin causes the top skin to deform, giving the corresponding morphed shape.

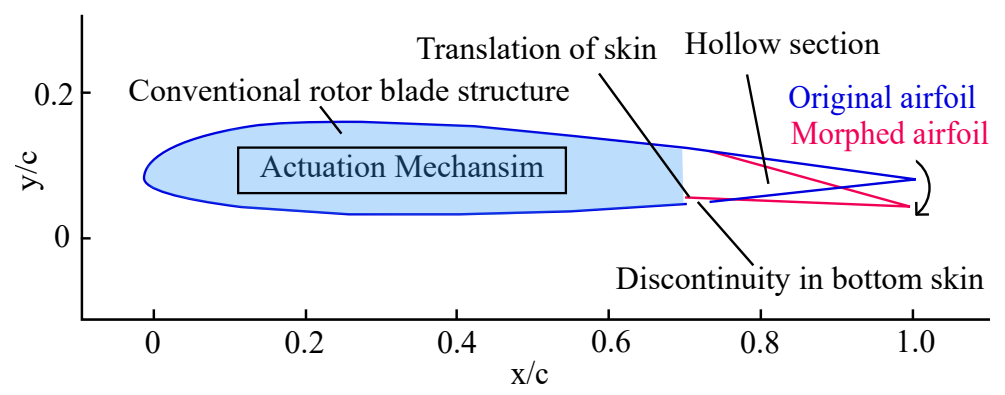

Fig. 2 Proposed operational principle of TRIC for rotorcraft.

In the active system, [21], the TRIC concept is actuated using an actuator that requires external energy to operate. The actuator is connected to the bottom surface using a mechanical link rod. The passive concept utilizes the same point for actuating the flap through the apparent centrifugal force. The novel concept [28] presented in this section is based on the operational requirements of the flap discussed by Vidyarthi et al. [22], where the morphing flap has to deflect downwards to its desired position between 100\% RPM and 90\% RPM. This required change in RPM is related to the corresponding change in the centrifugal force by the following expression:

$$
F_{c}=m r \omega^{2}
$$

where $m$ and $r$ represents the mass and distance of the centre of gravity (CG) of the rotating component respectively, $\omega$ indicates the rotor while $F_{c}$ represents the apparent centrifugal force. Accordingly, at a given rotational speed and neglecting the blade flapping angle, if a mass $\mathrm{m}$, comprising of a spanwise shaft and an extension spring, is placed within the rotor blade at a distance $r$ from the centre of rotation (COR), it experiences a centrifugal force normal to the velocity vector v, as shown in Fig. 3 and given by Eq. 1 .

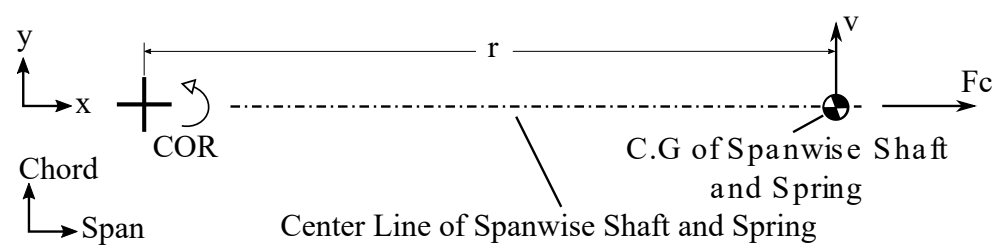

Fig. 3 Placement of the components w.r.t centrifugal force.

In view of Fig. 3, a mechanical arrangement in the form of orthogonal shafts is shown in Fig. 4 . The system of shafts shown in Fig. 4 converts the motion introduced by the apparent centrifugal force in the spanwise direction $\left(x_{s}\right)$ to a corresponding movement in the chordwise direction $\left(x_{t}\right)$ for the actuation of the flap. Extension spring with a 
preload $\left(p_{L}\right)$ is attached in line with the spanwise shaft such that any movement in the spanwise shaft causes the spring to extend. The preload in the extension spring ensures that the movement starts when the rotor RPM exceeds the $90 \%$ mark. The restoring force of the spring at 100\% RPM causes the flap to deflect downwards when the RPM is reduced to 90\%. The spanwise shaft (2), linked to the extension spring (4), provides an inclined surface for the chordwise shaft (1) to move. The relative movement of both the shafts is achieved by having rollers attached to the chordwise shaft (1) at the interface (6). The placement of the extension spring (4) and spanwise shaft (2) with respect to the overall system is shown in Fig. 3 It must be noted that, in principle, the shape of the spanwise shaft (2) at the interface (6) can be tailored for different flap deflection angles at various RPM. For instance, if the mission requirements need the flap angle to fluctuate between two positions with the change in RPM, the interface surface can be given a curved shape to achieve the desired behaviour. The interface connection can then be adapted accordingly.

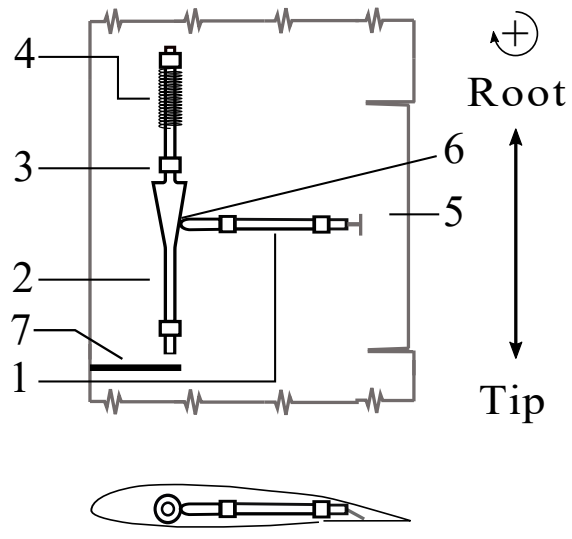

(a) Flap at $90 \%$ RPM
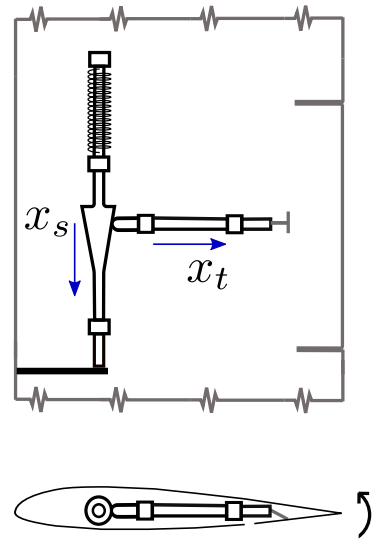

(b) Flap at $100 \%$ RPM

Fig. 4 Schematic of the passive system.

Figure 4 also shows the position of the flap with respect to the desired RPM value. When the rotorcraft is on the ground, and the rotor starts to spin, the flap deflects upwards to attain the undeflected or baseline position at 100\% RPM as shown in Fig. 4 (b). When the RPM decreases, the flap deflects downwards and attains the required deflection angle at $90 \%$ RPM as shown in Fig. 4(a). It must be noted that when the rotorcraft is about to take off, the collective pitch control is used to increase the rotor blade pitch angle. At this stage, the aerodynamic load can cause the flap to deflect upwards beyond its baseline position. To avoid this movement, it is important to have a mechanical endstop 7 shown in Fig. 4 that prevents the movement due to the aerodynamic load. When the flap is required to deflect downwards by the contraction in the extension spring, the reduction in the centrifugal force from 100\% RPM to $90 \%$ RPM must account for the aerodynamic load in order for the spring to contract as required.

The function of each component shown in Fig. 4 is summarized as follows:

1) Chordwise shaft (1) is linked to the trailing edge flap (5) at one end. The other end has roller bearings that are constrained inside the spanwise shaft (2). It is free to slide only in chordwise direction. 
2) Spanwise shaft (2) is placed perpendicular to the chordwise shaft (1), and its axis intersects the axis of the chordwise shaft (1). It provides a path for the roller bearings to move smoothly and also constrains the movement of the chordwise shaft (1). It is free to slide only in spanwise direction.

3) Extension spring (4) has a particular value of $p_{L}$ due to which it does not extend until the apparent centrifugal force exceeds that value as shown in Fig.5. It also enables the flap actuation at the desired RPM. The extension spring (4) is connected to the rotor blade structure at one end, and the other end is fixed to the spanwise shaft (2).

4) Support (3) refers to the mechanical housings for components such as mechanical sleeves that are fixed to the rotor blade structure. This results in constraining the movement of both the shafts in the desired direction while minimizing the friction.

5) Mechanical endstop (7) is attached to the rotor blade structure and limits the movement of the spanwise shaft (2) to ensure that the flap does not deflect upwards beyond its baseline position.

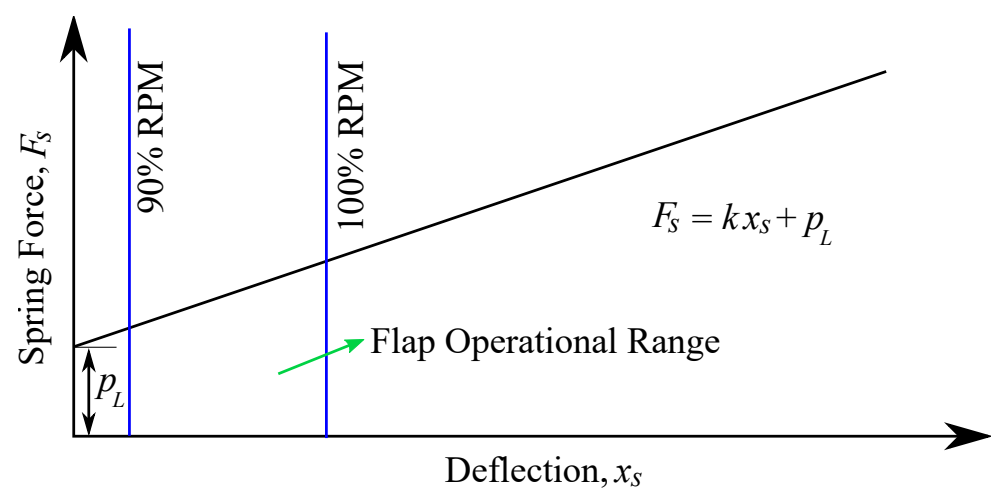

Fig. 5 Force and displacement relation of an extension spring.

\section{B. Design Parameters}

Based on the above-mentioned discussion, key design parameters are identified and presented in Fig. 6, The rotor parameters, which serve as the design inputs, include information such as flap length, flap location, flap chord, flap deflection angle, rotor radius, rotor blade airfoil and maximum RPM.

In a conventional flap with a distinct hinge, the aerodynamic load is the main force acting on the flap and the resulting hinge moment $H$ determines the actuation force $F_{a}$. However, in the TRIC morphing flap, the elastic load is also a key design input, and an actuation force $F_{e}$ is required to compensate it. This elastic load results from the stiffness of the flexible morphing skin and depends on the size of the flap, its thickness, and material. Therefore, to design a passive system around such a concept, both the elastic and aerodynamic loads must be determined. Once these two loads are known, the net force $F_{N e t}$ is calculated against which the centrifugal force has to operate. The other important parameters which are needed to be determined include the stroke or chordwise movement $x_{t}$ of the bottom skin required for the desired flap deflection $\beta$. This chordwise movement is linked to the spanwise movement $x_{s}$ of 


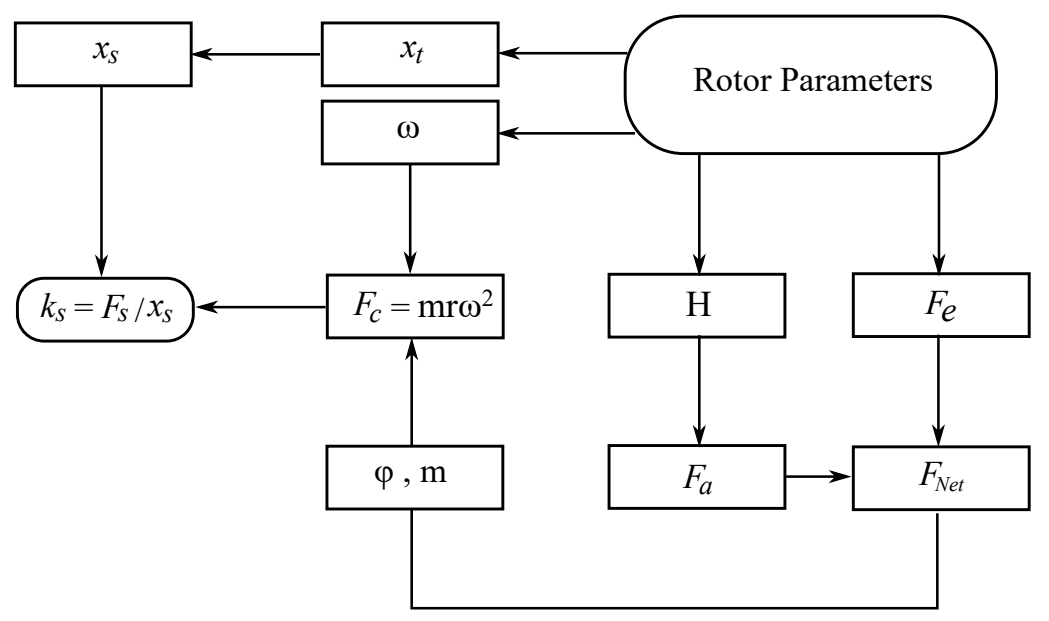

Fig. 6 Block diagram representing the design parameters.

the extension spring. The shaft angle $\varphi$ resolves the net force required to deflect the flap into spanwise and chordwise components. However, it must be noted that in principle, the shape of the spanwise shaft (2) at the interface (6) can be tailored for different flap angles at various RPM. After having all the input parameters identified, the required spring stiffness $k_{s}$ and the $p_{L}$ of the extension spring can be estimated.

Figure 6 indicates that the design of the passive system involves the estimation of the aerodynamic forces and elastic forces. These forces can be determined either by doing high fidelity analysis using Finite Element Analysis (FEA) and Computational Fluid Dynamics (CFD) or by analytical formulations using the classical beam theory [29], the Classical Laminate Theory (CLT) [30] and blade element theory for hovering, assuming uniform inflow and symmetric, untwisted rotor blades [31].

\section{Passive System Demonstrator and Test Setup}

For the proof-of-concept of the passive camber morphing system, a demonstrator is designed, which is presented in the form of a CAD model in Fig. 7. The outer geometry is given an aerodynamic shape considering NACA 23012 with a chord length of $270 \mathrm{~mm}$. In the proposed mechanism, the spanwise shaft is supported by two flanges at each end having steel bushes. One end of the extension spring is connected to the spanwise shaft while the other end is fixed to the beam. The centre portion of the spanwise shaft is tapered, providing an inclined surface for the rollers. These rollers, which are attached to the chordwise shaft, are secured inside the groove provided by the spanwise shaft. The other end of the chordwise shaft is connected to a male rod end which is attached to the metal bracket bonded to the flap. This shaft is also supported by bearing support and pin support, as shown in Fig.7.

The manufactured model with the two main shafts is shown in Fig. 8 . In this figure, the groove and path for roller bearings can be seen. It is also worth mentioning here that the initial position of the flap is achieved by adjusting the length of the chordwise shaft appropriately. 


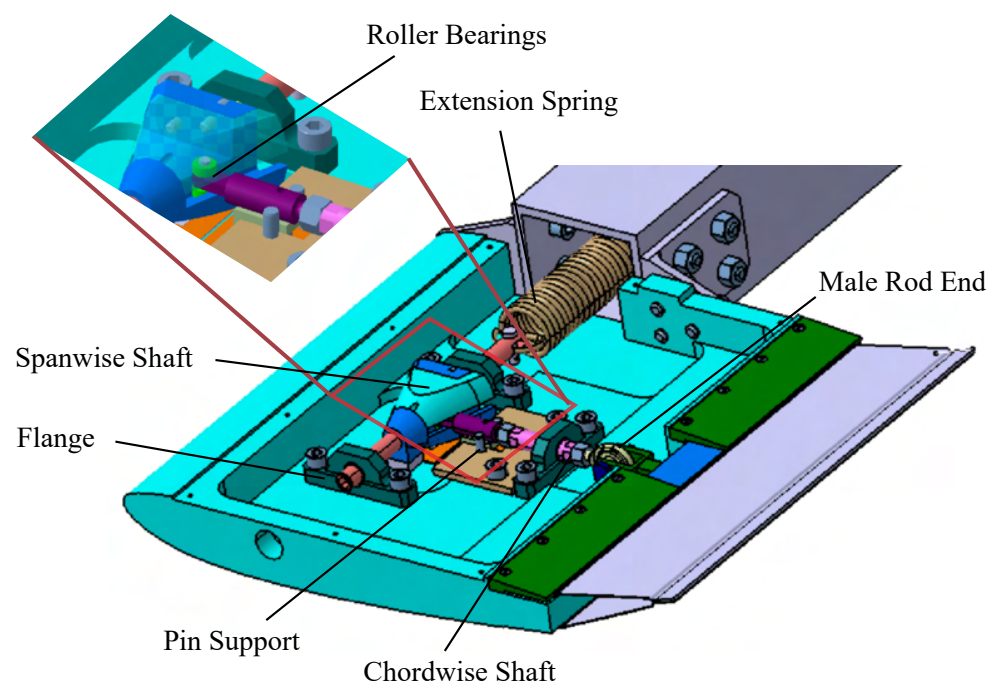

Fig. 7 A detailed CAD model of the passive system.

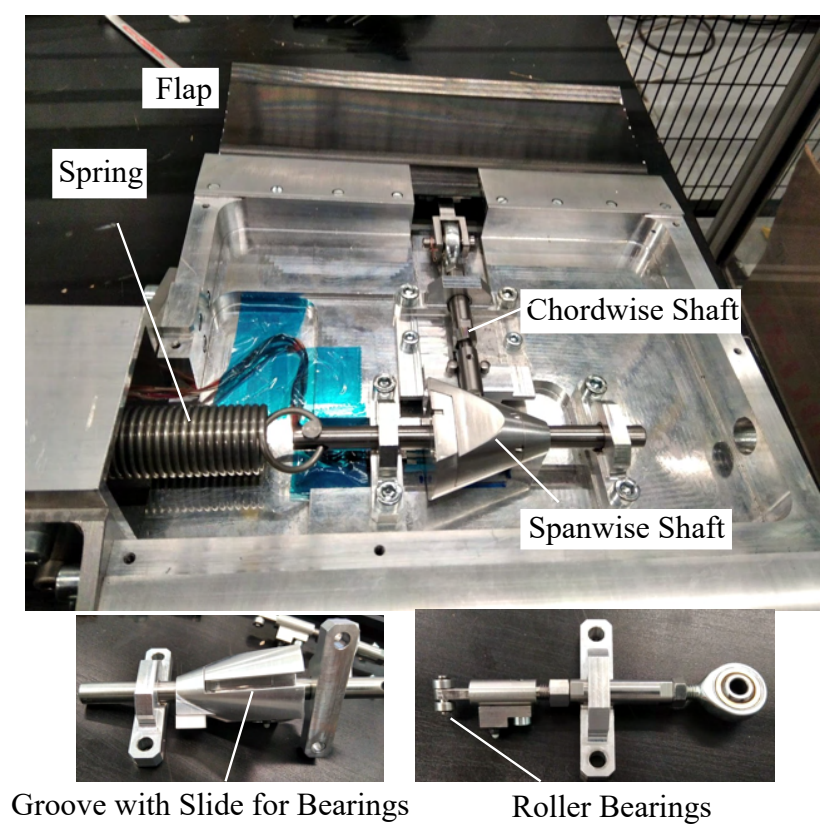

Fig. 8 Physical model of the passive system.

The validation of the passive system is carried out on a whirl tower test setup which can rotate at a maximum speed of 240 RPM. The details of this whirl tower test setup are presented in Ref. [21]. However, before taking the passive system to the whirl tower test, a bench test is performed using a pulley and string arrangement as shown in Fig. 9. This setup is used to estimate the initial tension in spring and the force required to deflect the flap, as shown in Fig.9 (a). In addition, the same setup is utilized to estimate the force required by a spring to hold the flap at its deflected initial position. This is accomplished by removing the spring from the assembly and attaching a string with suitable weights hanging from a pulley, as shown in Fig.9(b). 


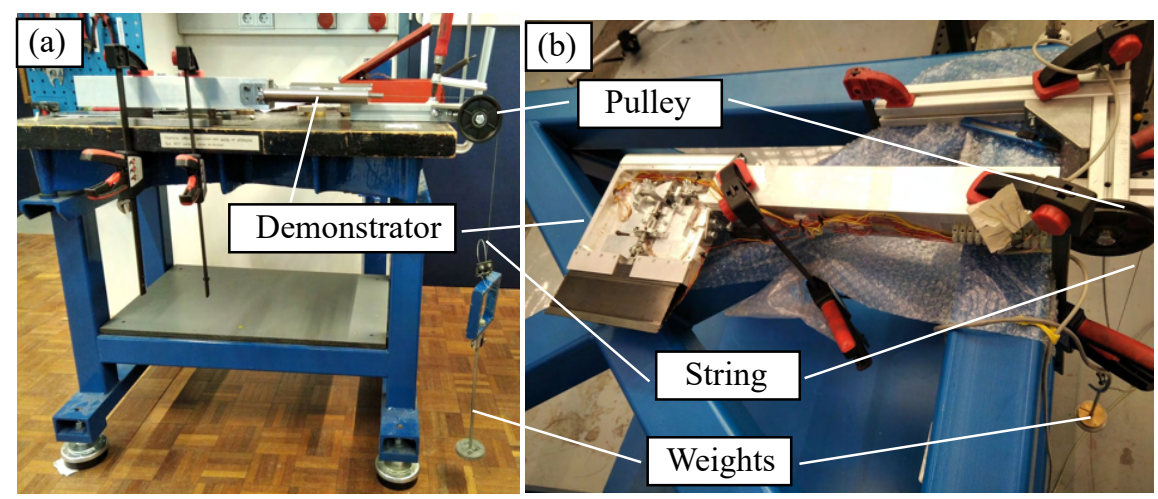

Fig. 9 Bench test setup: Functional test (a), Flap elastic force (b).

A detailed CAD model of the complete demonstrator for the passive morphing system is shown in Fig. 10 This model is developed with the consideration that it is balanced properly on the whirl tower test setup and that the CG of the spanwise shaft and spring assembly lies on the x-axis (spanwise direction) without any chordwise offset as shown in Fig. 3.

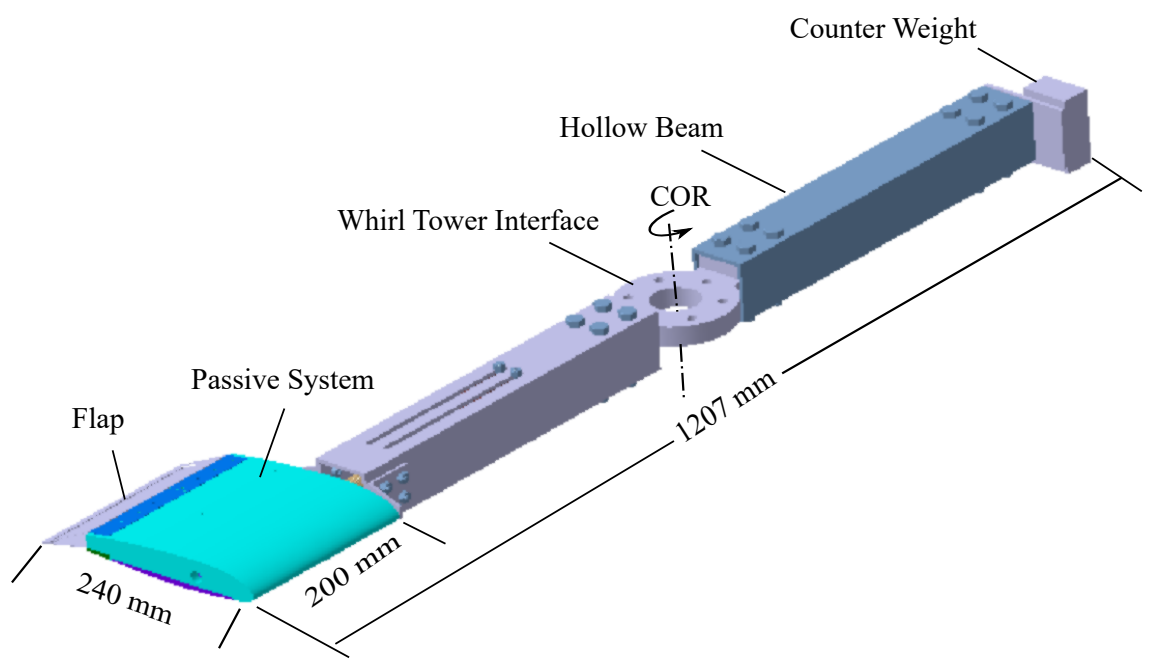

Fig. 10 Passive demonstrator for whirl tower test.

In total, five springs are used for the test campaign, with variations in $p_{L}, k_{s}$ and weight. These springs are selected based on the maximum allowable RPM permitted on the whirl tower setup. Since initial tension and the spring constant are the two driving parameters for the selection of the spring for the passive system, both these parameters are obtained experimentally for all the springs using a tensile test machine as shown in Fig.11.

The arrangement for the passive whirl tower test is shown in Fig.12 The passive morphing experiment is conducted mainly for the validation of the concept using different springs. The main objective of this test campaign is to develop an understanding of the behaviour of the passive system and to monitor the spring deflections and the corresponding flap tip deflections. By using different springs in the process, the consistency of the passive system is demonstrated. In 


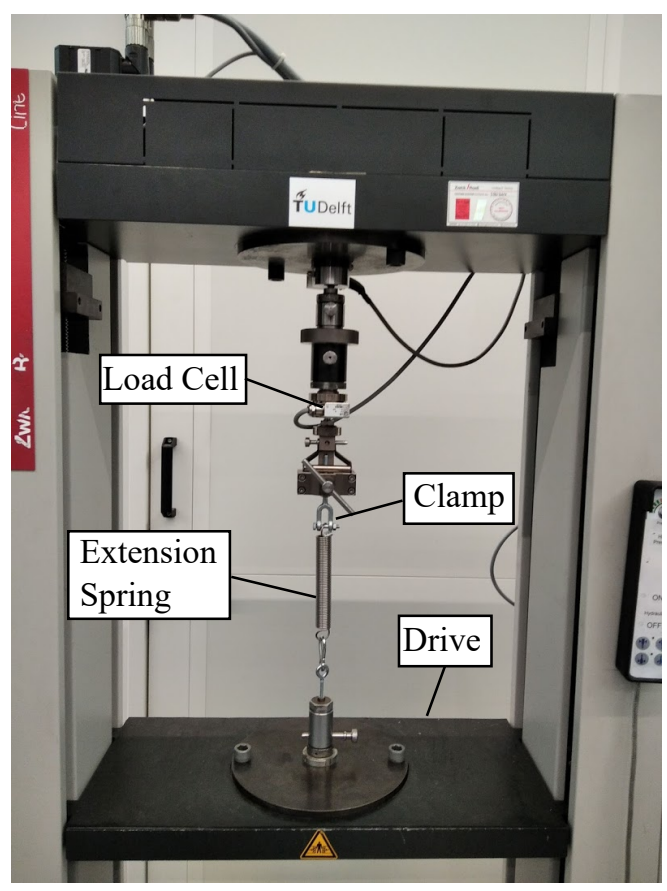

Fig. 11 Spring test for initial tension and spring constant.

this test, the focus is to obtain the spanwise and chordwise movements of spanwise and chordwise shafts, respectively, and also the corresponding tip deflections of the flap. A displacement transducer (DT) is fixed underneath the spanwise shaft and chordwise shaft, providing the stroke measurement a spring-loaded plunger moves over it. For the flap tip deflection, a high-speed camera is used. In order to capture the tip displacement of the flap from the high-speed camera, a single LED is placed on the tip of the flap to indicate its deflection. In addition, one LED is set as a fixed point (FP) attached to the ground while four LEDs are placed on the aluminium housing to indicate any torsional deformations of the demonstrator. These four LEDs are on the same plane. The arrangement of LEDs with their respective positions is shown in Fig. 13 .

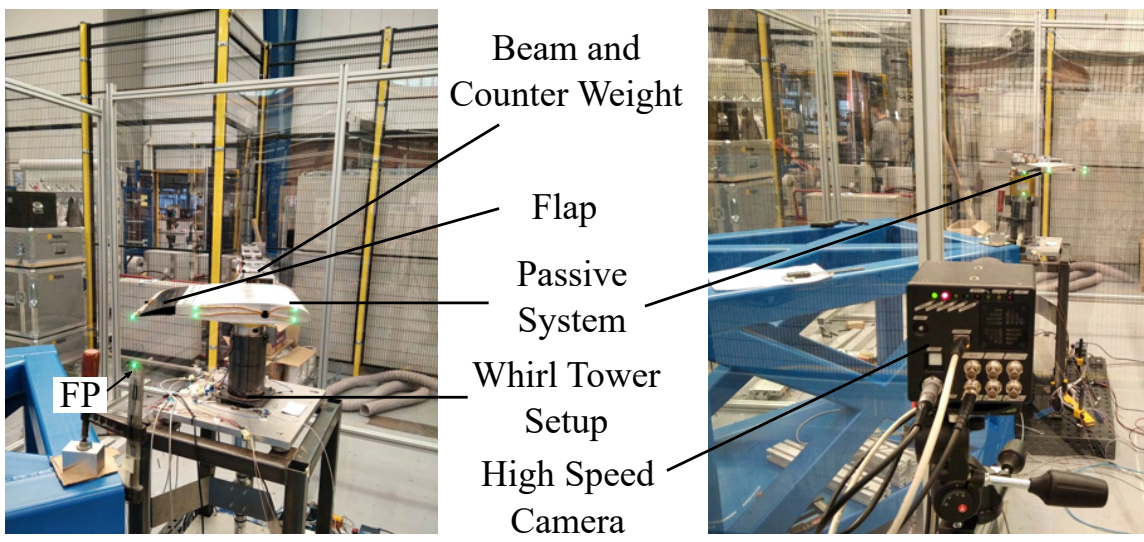

Fig. 12 Whirl tower setup for testing the passive system. 


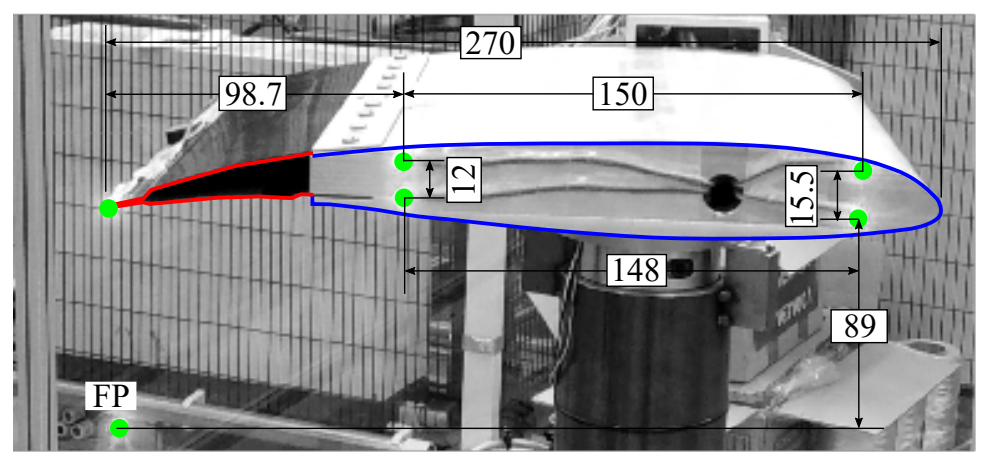

Fig. 13 Placement and position (in mm) of LEDs on the demonstrator.

\section{Whirl Tower Test Results}

In this section, the results of the experimental campaign with the passive system are discussed. As mentioned in Sec. [V] the springs in the passive system are tested for the preload and $k_{s}$ using a tensile test machine. The deflection of each spring against the applied force is shown in Fig. 14. From the deflection curves shown in Fig. 14, the consolidated data is provided in Tab. 1 including the specification of each spring provided by the original equipment manufacturer (OEM).

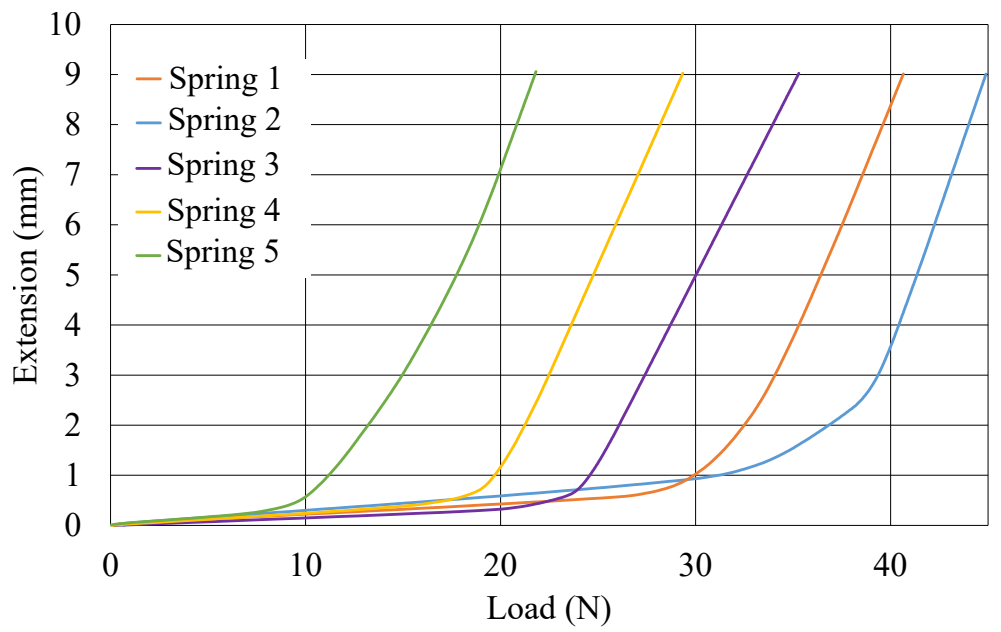

Fig. 14 Spring extension under axial load.

It can be observed from Fig. 14 that all the springs show a non-linear region until the spring preload is overcome. This behaviour can be attributed to the deflection of the end loops [32]. The springs exhibit a linear response once the preload is completely overcome. The spring constants shown in Tab. 1 are obtained by taking the linear portion of the curves in Fig. 14 between the extensions of $3 \mathrm{~mm}$ and $9 \mathrm{~mm}$ and then taking the slope of these curves. Figure 14 also shows that for all the springs, it is not possible to precisely specify the value of preload. The reason for not having a specific value of preload is because the deflection is not exactly zero for a particular value of force; rather, it has an 
Table 1 Specification of springs.

\begin{tabular}{cccccc}
\hline & & \multicolumn{2}{c}{ Measured } & \multicolumn{2}{c}{ OEM } \\
\cline { 3 - 6 } ID & Mass $(\mathrm{g})$ & preload $(\mathrm{N})$ & $\mathrm{Ks}(\mathrm{N} / \mathrm{mm})$ & preload $(\mathrm{N})$ & $K_{s}(\mathrm{~N} / \mathrm{mm})$ \\
\hline Spring 1 & 80.97 & 25 & 1.1 & 25.66 & 1.22 \\
Spring 2 & 132.95 & 20 & 0.92 & 22.66 & 0.82 \\
Spring 3 & 65.38 & 22.7 & 1.31 & 22 & 1.18 \\
Spring 4 & 71.58 & 18 & 1.14 & 22 & 1.05 \\
Spring 5 & 80.65 & 10 & 1.13 & 22 & 0.94 \\
\hline
\end{tabular}

approximate value of $0.5 \mathrm{~mm}$ (except Spring 2), after which the linear portion of the curve starts. The measured preload mentioned in Tab. 1 1 is calculated against the deflection of $0.5 \mathrm{~mm}$ for all the cases. It must be noted that Spring 2 shows more non-linear behaviour at the beginning, and the linear part of the curve starts at $2 \mathrm{~mm}$ of deflection approximately. The aforementioned discussion concerning the spring data is important as the passive system is also expected to behave in a similar way. For instance, let us consider that two identical springs are selected on the basis of preload and spring stiffness. The first spring shows a higher nonlinearity in terms of its stiffness than the second spring at the beginning of its extension. This would mean that to achieve the required deflection with the first spring; more force would be required to overcome the spring force. On the other hand, the spring which shows a relatively linear stiffness from the start would attain the desired deflection with a lesser applied force. For a passive system, this means employing a different maximum RPM value in both the aforementioned cases to have a similar flap deflection.

Another critical aspect of passive morphing testing is the amount of friction observed in the manufactured prototype. During the initial bench tests, it is observed that the amount of static and kinetic friction present in the system is so high that no flap movement is observed in the region of interest where the RPM is dropped from $100 \%$ to $90 \%$. This behaviour is shown in Fig. 15 where the loading and unloading cycles are plotted for the case when the system is integrated with Spring 3 spring, and no mechanical refinement is carried out. The plots represent the movement of the spanwise shaft against the applied load obtained using a DT. In the loading cycle, the weights are loaded in small steps to record the spring extension. During the unloading cycle, the weights are removed, and the corresponding movement of the spring is recorded. It can be seen in Fig. 15 that even after removing the load below $90 \%$ of the maximum applied load, the restoring force exerted by the spring is less than the maximum static force of friction. Similar behaviour is seen at the start of the loading curve, where the onset of deflection is delayed considerably. After observing the issue, considerable effort is put to reduce the excessive amount of friction by using suitable lubricants and adjusting the bearing supports of the two shafts by using very thin shims to improve the shaft alignment. Although the friction is not completely removed from the system in the end, the mechanical refinements improved the functionality of the passive system considerably, as shown in Fig. 15. After the mechanical refinements, the unloading cycle caused the flap to deflect downwards within the range of interest, indicating the working of the passive concept. It has to be noted that the 
geometrical discrepancies and shaft misalignment are issues that are expected in a proof-of-concept demonstrator and can be improved in future models.

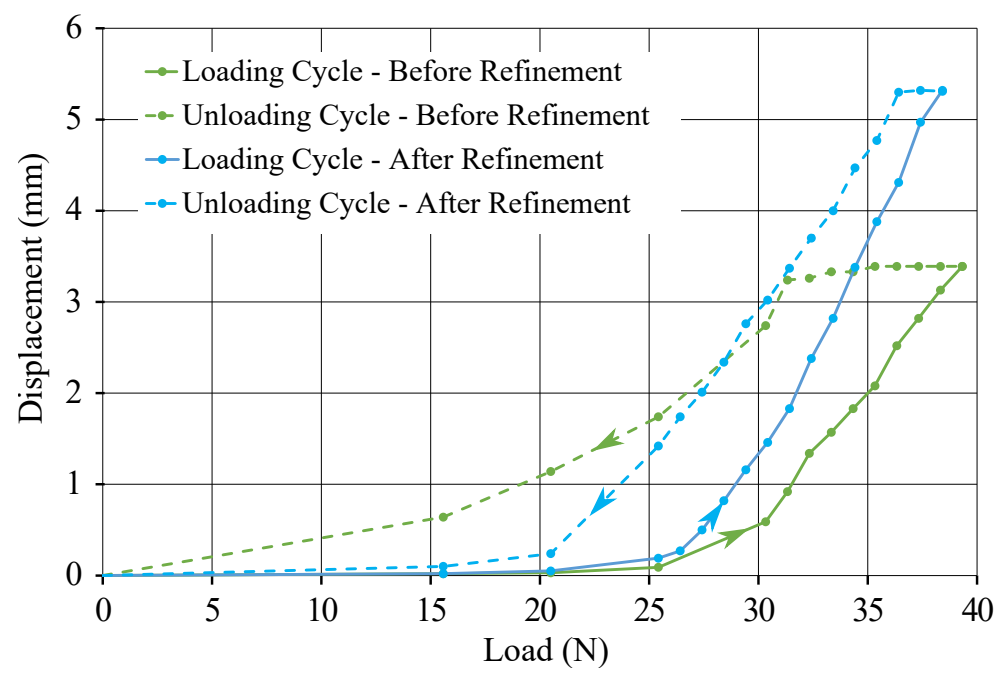

Fig. 15 Effect of mechanical refinement on kinetic friction.

Before carrying out the experiments of the complete passive system on the whirl tower setup, tests with each spring are performed on the setup with flap disengaged. The results corresponding to each extension spring are plotted in Fig. 16 and show a similar trend for each spring when compared to the curves in Fig. 14. In Fig. 16, however, the influence of friction in the system even with a single shaft is prominent in all the cases, emphasizing the importance of location, alignment, concentricity, surface finish, bearing selection, and geometrical tolerances. All these factors have to be addressed during the future design, manufacturing, and integration of the passive system to improve its performance.

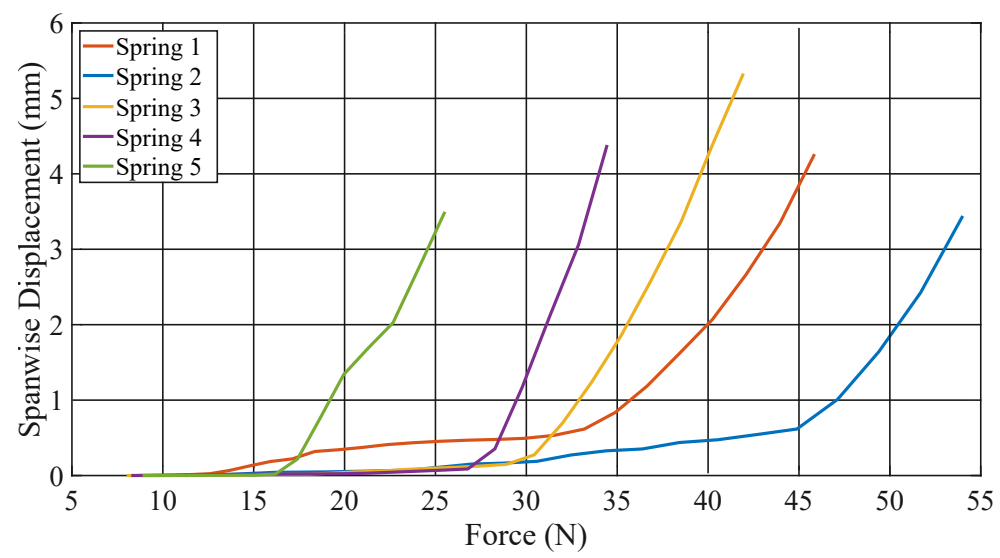

Fig. 16 Measurement of spanwise displacement without flap under centrifugal load.

With reference to the plots in Fig. 16, Spring 1 shows slightly different behaviour at the onset of deflection. This behaviour is attributed to the small freeplay present at the point of connection of the spring and the spanwise shaft as 
shown in Fig. 17. The corresponding load extension curve shows a slightly larger offset at the beginning, which is due to the small displacement that occurs between $15 \mathrm{~N}$ and $20 \mathrm{~N}$ due to the freeplay. Afterwards, the curve is flattened due to the preload of the spring till the point where the spring starts to deflect. This indicates that any freeplay present in the system can affect the behaviour of the passive system.

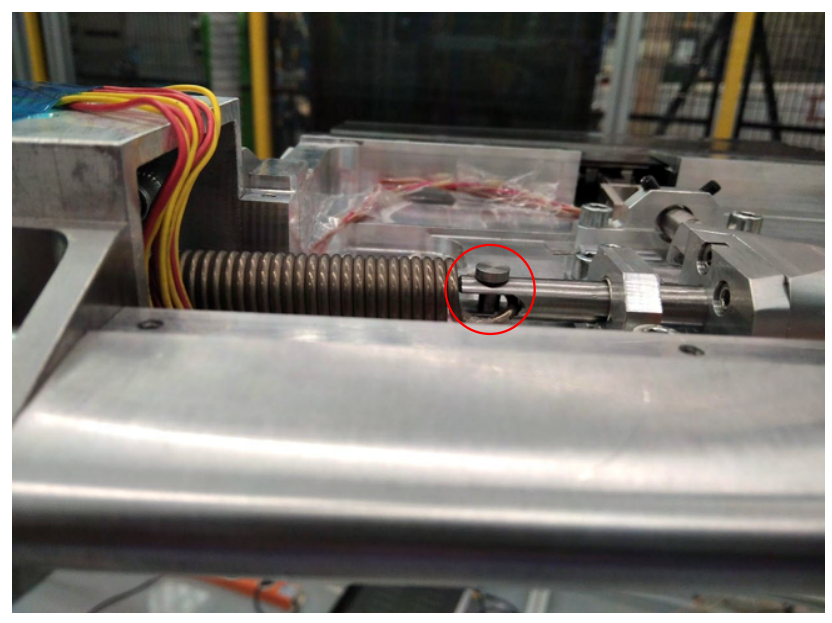

Fig. 17 Closeup of the freeplay in the spring and connecting pin.

Also, for the case of Spring 2 shown in Fig. 16, the system under centrifugal loads shows a similar behaviour as shown in Fig. 14 The onset of the linear portion of the load-deflection curve is delayed more due to the higher non-linear region at the start. The relatively linear portion of the loading cycle is observed around $2 \mathrm{~mm}$ of deflection.

After doing the initial tests, the complete passive system is tested with all the springs on the whirl tower test setup. The 100\% RPM mark is varied based on the preload of each individual spring till a spring extension of approximately 6 $\mathrm{mm}$ is achieved for each case. As a sixth case, a small trimming mass is attached in front of the spanwise shaft, and the test is performed with one spring, i.e., Spring 1 to observe the effect of added mass in the system. The weight of the resulting mass is dictated by the space and attachment constraints and turned out to be $57 \mathrm{~g}$. Since the whirl tower is operated independently of the data acquisition system, it is not possible to automatically synchronize the spring deflection and the RPM. For this reason, tests are performed initially for all the spring cases, and the region of the desired flap deflection is observed. This resulted in determining the particular RPM value, which brought the springs to their mean positions and enabled establishing the $90 \%$ and 100\% RPM mark for each case. Afterwards, the data is recorded for this RPM range, where the overall deflection of the spring is captured using the DT, and the tip deflection of the flap is recorded using the high-speed camera.

Figure 18 shows the overall response of the system with Spring 1, including the tip deflection. The centrifugal force plotted on the $\mathrm{x}$-axis in Fig. 18a, corresponds to a particular RPM value, and it is calculated using Eq. 1. where $\mathrm{m}$ accounts for the total mass of the spring and the shaft, and $r$ is taken as the sum of the distance of the centre of gravity 
of $\mathrm{m}$ from the centre of rotation and extension of the spring. The value of $\mathrm{r}$ is estimated from the CAD model of the passive system for each case. In Fig. 18a the spanwise movement of the system is plotted for the four phases of the movement of the spanwise shaft connected to the extension spring. This division in four phases is important to establish since it is related to the operational scenario of the passive system and serves to show its complete functional cycle. In the first phase (shown as black curve), the RPM is increased from 150 to 240, and the corresponding displacement is recorded at steps of 5 RPM. The increase in RPM is accomplished manually with steps of 1 RPM due to the limitation of the whirl tower setup. In the second phase (shown as dark blue curve), the speed is reduced gradually to 216 RPM, which is the $90 \%$ mark. In the third phase, the speed is again increased to 100\% RPM (shown as the green curve) and then dropped to 150 RPM (shown as the cyan curve) in the fourth phase. Considering the four phases mentioned, the flap tip displacement is captured using a high-speed camera. The recorded images are post-processed to obtain the position of the flap tip with reference to the LED, which is fixed to the ground. Consequently, the flap tip deflection is obtained at each stage corresponding to a particular RPM as indicated in Fig. 18a and shown in Fig. 18b. The four points attached to the aluminium structure are also plotted for each case as shown in Fig. $18 \mathrm{~b}$ A consistent overlap of respective points indicates that the structure does not undergo any torsional behaviour during the testing. The vertical axis in Fig. $18 \mathrm{~b}$ shows the position of the flap with respect to the FP for each stage of the operation. The horizontal axis shows the position of the LEDs in the chordwise direction. Similar tests are performed for the remaining springs and shown in Fig. 19 to Fig. 22

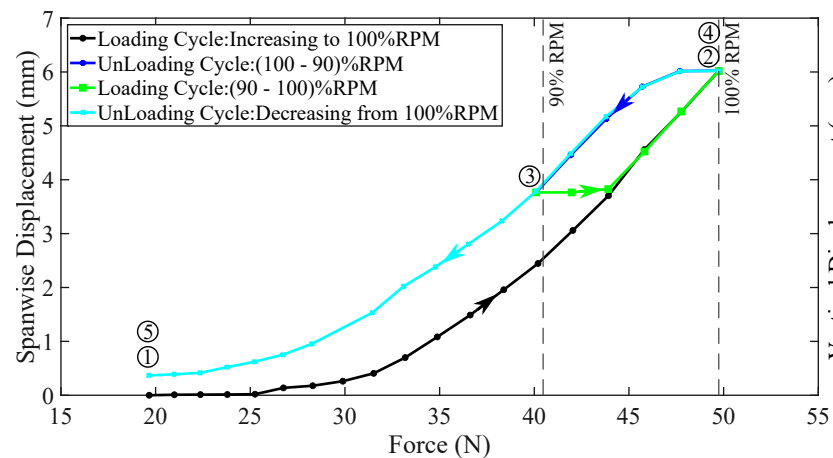

(a) Spanwise displacement.

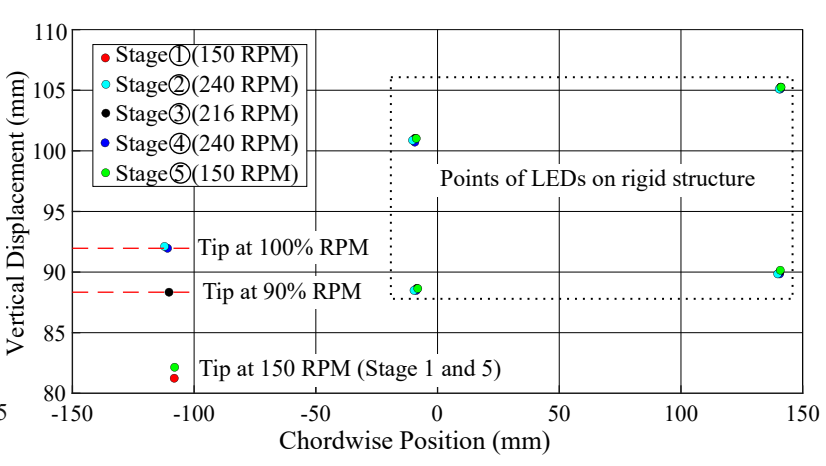

(b) Flap tip deflection.

Fig. 18 Measurements with Spring 1 with RPM variation from 150 to 240.

Due to the difference in specifications, the RPM limits are different for each spring, and thus the operating range is also different. However, having an approximately similar displacement of the spanwise shaft, the data plotted in Fig. 18 to Fig. 22 gives some important insight into the response of the passive system. From the measurements presented for each spring, it can be observed that the response of the passive system is directly linked to the preload and stiffness of the individual springs. Furthermore, the available force in the window of operation defined by the 90\% RPM and $100 \%$ RPM is critical for the desired flap deflection, and it is influenced by the combined mass of the spanwise shaft and the 


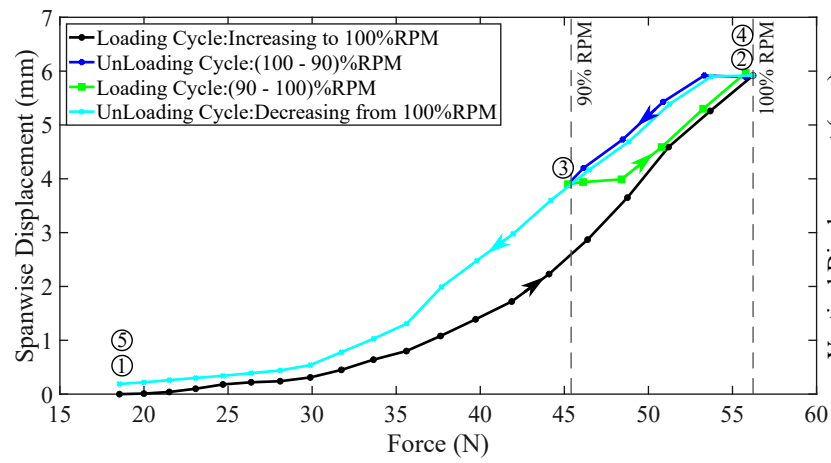

(a) Spanwise displacement.

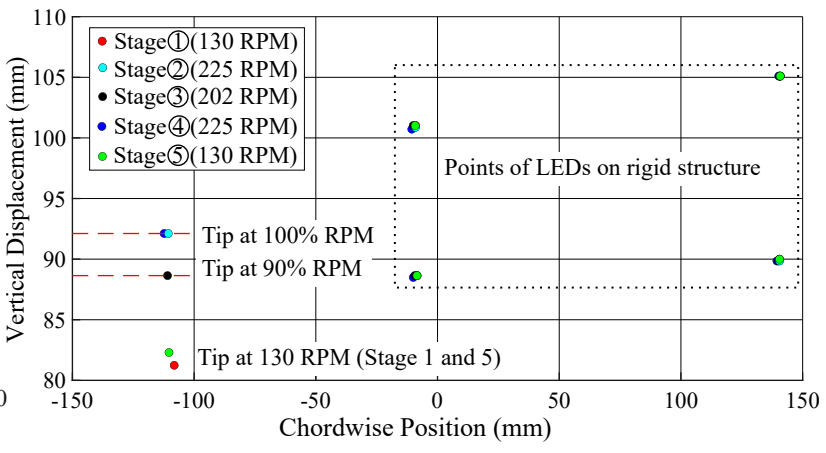

(b) Flap tip deflection.

Fig. 19 Measurements with Spring 2 with RPM variation from 130 to 225.

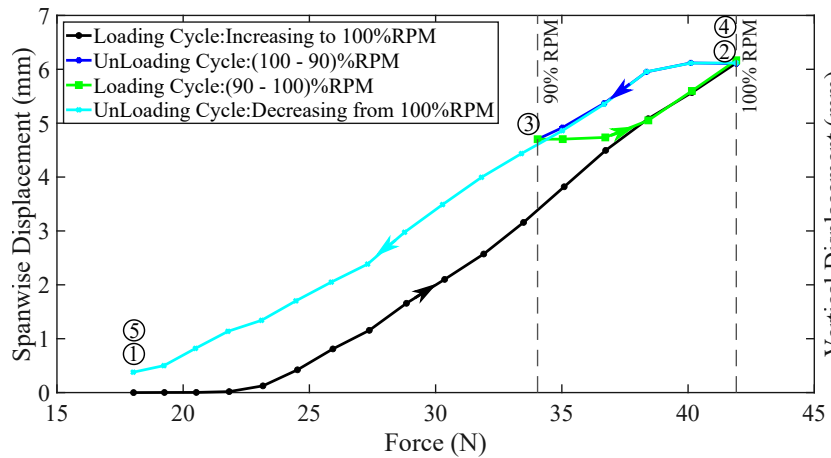

(a) Spanwise displacement.

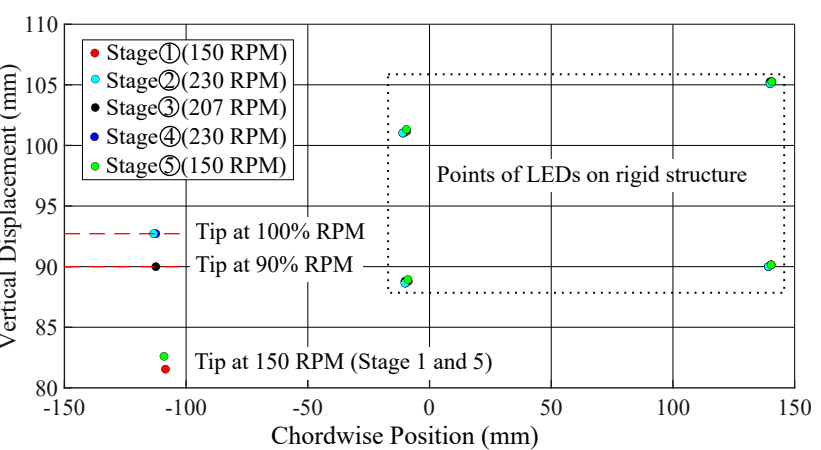

(b) Flap tip deflection.

Fig. 20 Measurements with Spring 3 with RPM variation from 150 to 230.

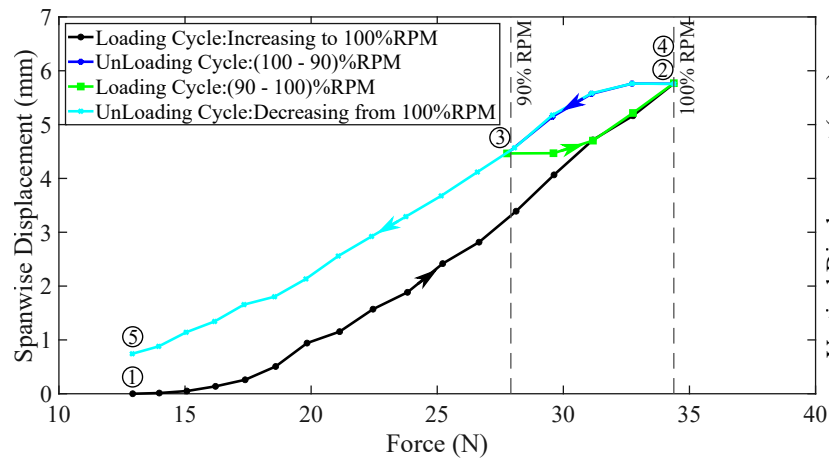

(a) Spanwise displacement.

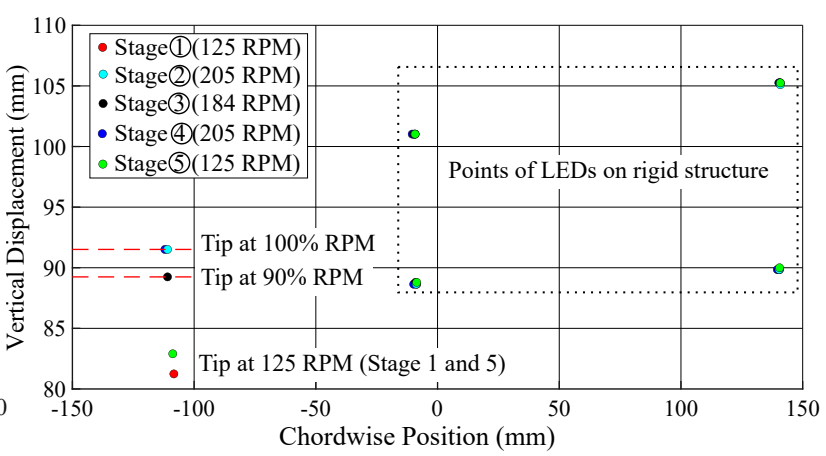

(b) Flap tip deflection.

Fig. 21 Measurements with Spring 4 with RPM variation from 125 to 205.

spring. In addition, it can also be observed from the plots that more net force is available to the spring between $90 \%$ RPM and 100\% RPM window if the maximum value of RPM is higher.

Figure. 18a indicates that Spring 1, which has the highest value of the preload, shows the highest value of extension in the 90\% RPM to 100\% RPM range. Consequently the flap tip deflection shown in Fig. 18b] is also the highest. The delay in the onset of the linear portion of the unloading cycle shown in Fig. 18a indicates the amount of static friction 


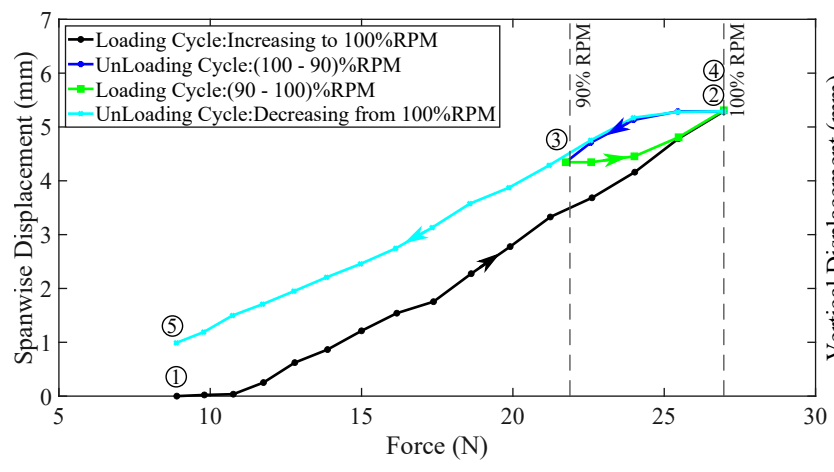

(a) Spanwise displacement.

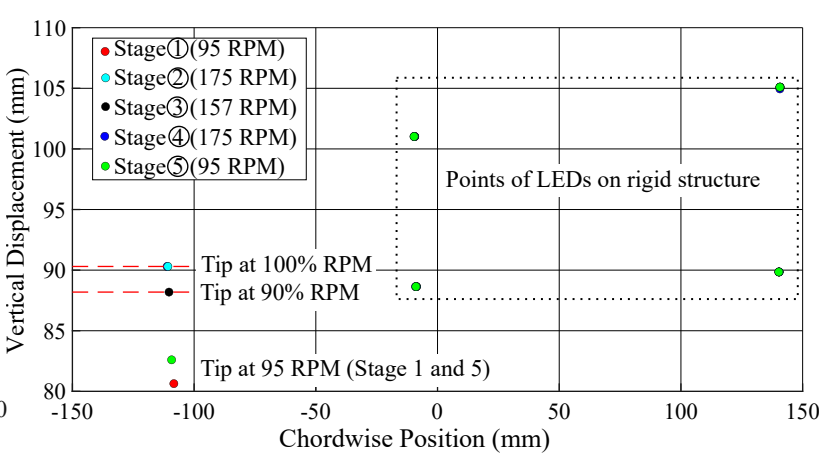

(b) Flap tip deflection.

Fig. 22 Measurements with Spring 5 with RPM variation from 95 to 175.

present in the system that must be overcome before the movement starts.

In the case of Spring 2, the maximum value of RPM is observed, which subsequently gives rise to the maximum centrifugal force. The main reason for the higher centrifugal force in the case of Spring 2 observed in Fig. $19 \mathrm{a}$ is due to the nonlinearity of the spring discussed earlier. Due to the higher stiffness value at the initial stage, more force is required to extend the spring to the desired value, resulting in a higher RPM value in the end. The deflection measured for the Spring 2 is very close to the Spring 1, as shown in Fig. 19b

For the remaining springs, the response of the system is mainly dictated by the preload as shown in Fig. 20, Fig. 21 and Spring 5 has the least amount of net force available within the 10\% RPM range to deflect. This is because this particular spring has the lowest preload. Consequently, the amount of tip deflection is also minimum, as shown in Fig. $22 \mathrm{~b}$ The role of preload is noticeable in all the cases due to the difference in values mentioned in Tab. 1 . On the contrary, the effect of stiffness cannot be detected in this study due to approximately similar values of stiffness of all the springs.

For the last case, the effect of increasing the weight of the spanwise shaft is estimated by attaching a trimming weight resulting in an increase of $35 \%$ in the effective mass. The trimming weight is attached in the form of a rod which is screwed at the end of the spanwise shaft. The corresponding result of the passive system with the new configuration is shown in Fig. 23. It can be seen that the RPM for similar deflection is reduced considerably by the addition of the trimming mass. The net force between $90 \%$ and $100 \%$ for Spring 1 is approximately the same with and without the trimming mass. However, the RPM range for the two cases is considerably different. Due to this reason, reduction in the $100 \%$ RPM by 5 RPM accounts for a greater force with the trimming mass than for the same amount of RPM reduction without the trimming mass. The aforementioned trend is noticeable in the unloading curves for both cases shown in Fig. $18 \mathrm{a}$ and Fig. $23 \mathrm{a}$

The contribution of the flap is also apparent in all the measurements when compared to Fig. 16. This is also important since the aerodynamic loads on the flap are expected to be considerably low because of the size of the flap, 


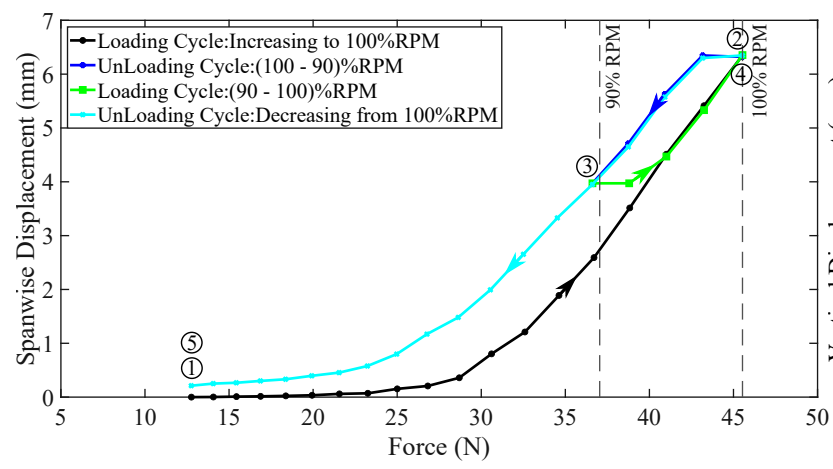

(a) Spanwise displacement.

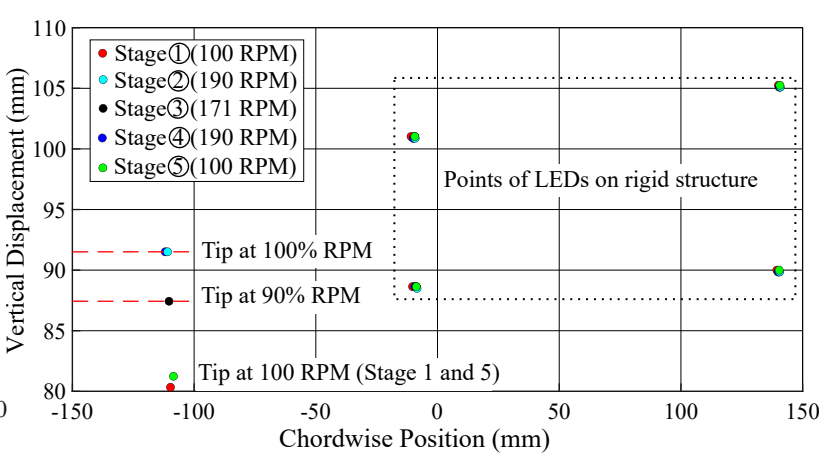

(b) Flap tip deflection.

Fig. 23 Measurements with Spring 1 and trimming weight and with RPM variation from 100 to 190.

the radius of the demonstrator and the maximum allowable RPM. It can be noticed that, effectively, spring preload reduces with the introduction of the flap for all the cases.

\section{Conclusions}

In this paper, the design and experimental validation of a centrifugal-force-operated concept are presented for a VSR. For the case of hover, such a passive system can potentially increase the pilot control authority and reduce the power requirement by $8 \%$. Further studies are required to observe the effect of passive camber morphing in forward flight. To achieve the potential benefits in hover, a novel concept is introduced to achieve the desired camber morphing of the rotor blades by varying the rotor RPM. The designed morphing mechanism successfully achieved the desired morphing configurations by changing the RPM of the rotor. Moreover, the mechanism can be tailored based on RPM and morphing requirements.

The validation of the passive system was carried out in a whirl tower test setup. From the extensive testing, one can conclude that the novel passive system operates within the prescribed RPM range of 10\%. The experiment was repeated with various springs having different preloads and stiffness values. In each case, it was observed that the flap started to deflect towards the baseline position once the apparent centrifugal force exceeded the preload in the spring. When the RPM was reduced to the $90 \%$ mark, the flap deflected downwards depending on the restoring force of the spring. This trend was indicative of a consistent behaviour of the passive system. However, the mechanical performance of the mechanism was affected, due to which the extension in the spring and the corresponding tip deflection of the flap turned out to be lower than expected. It is clear that once the rotor starts to spin, the upward flap deflection depends on the spring preload that keeps it deflected initially. For full-scale helicopters such as Bo-105, which have the main rotor spinning at around 400 and 450 RPM, this implies using extension springs with considerably large values of preload.

Despite the friction in the mechanism, the system provides opportunities for further investigations. The friction in the demonstrator consumes a significant amount of energy and signifies the importance of careful considerations 
during the design, manufacturing, and integration stages. For instance, the mechanism can be manufactured to greater precision in future. In addition, the option of re-designing the mechanism which is less sensitive to manufacturing and integration accuracies can also be explored.

\section{References}

[1] Amendola, G., Dimino, I., Concilio, A., Pecora, R., Amoroso, F., and Arena, M., Morphing Aileron, Elsevier Ltd., 2017.

[2] Noboru, K., Saito, S., Fukami, T., and Komura, T., "Design and performance evaluation of full scale on-board active flap system," Annual Forum Proceedings - AHS International, Vol. 1, 2007, pp. 212-222.

[3] Delrieux, Y., Le Pape, A., Leconte, P., Crozier, P., Gimonet, B., and Des Rochettes, H. M., "Wind-tunnel assessment of the concept of active flaps on a helicopter rotor model," Annual Forum Proceedings - AHS International, Vol. 3, 2007, pp. $2297-2310$.

[4] Oktay, T., and Sal, F., "Combined passive and active helicopter main rotor morphing for helicopter energy save," Journal of the Brazilian Society of Mechanical Sciences and Engineering, Vol. 38, No. 6, 2016, pp. 1511-1525.

[5] Army, U., Rotary Wing Flight - Flight Manual 1-51, United States, Department of the Army, 1979.

[6] Han, D., and Smith, E. C., "Lagwise dynamic analysis of a variable speed rotor," Aerospace Science and Technology, Vol. 29, No. 1, 2013, pp. 277-286.

[7] Amri, H., Feil, R., Hajek, M., and Weigand, M., "Possibilities and difficulties for rotorcraft using variable transmission drive trains," CEAS Aeronautical Journal, Vol. 7, No. 2, 2016, pp. 333-344.

[8] Horn, J. F., "Implementation of Variable RPM in Helicopter Flight Control Laws," The 3rd International Basic Research Conference on Rotorcraft Technology, 2009.

- [9] Misté, G. A., and Benini, E., "Variable-Speed Rotor Helicopters: Performance Comparison Between Continuously Variable and Fixed-Ratio Transmissions," Journal of Aircraft, Vol. 53, No. 5, 2016, pp. 1189-1200.

[10] Gustafson, F. B., and Gessow, A., "Effect of rotor-tip speed on helicopter hovering performance and maximum forward speed," Naca Arr No. L6a16, 1946.

[11] Silva, C., Yeo, H., and Johnson, W., "Design of a Slowed-Rotor Compound Helicopter for Future Joint Service Missions," American Helicopter Society Aeromechanics Specialists' Conference, 2010.

[12] Floros, M. W., and Johnson, W., "Performance Analysis of the Slowed-Rotor Compound Helicopter Configuration," Journal of the American Helicopter Society, Vol. 54, No. 2, 2009.

[13] Han, D., and Barakos, G. N., "Variable-speed tail rotors for helicopters with variable-speed main rotors," Aeronautical Journal, Vol. 121, No. 1238, 2017, pp. 433-448. 
[14] Xie, J., Guan, N., Zhou, M., and Xie, Z., "Study on the Mechanism of the Variable-Speed Rotor Affecting Rotor Aerodynamic Performance,” Applied Sciences, Vol. 8, No. 7, 2018.

- [15] Han, D., Pastrikakis, V., and Barakos, G. N., "Helicopter performance improvement by variable rotor speed and variable blade twist," Aerospace Science and Technology, Vol. 54, 2016, pp. 164-173.

[16] Barbarino, S., Gandhi, F., and Webster, S. D., "Design of extendable chord sections for morphing helicopter rotor blades," Journal of Intelligent Material Systems and Structures, Vol. 22, No. 9, 2011, pp. 891-905.

[17] Khoshlahjeh, M., and Gandhi, F., "Extendable chord rotors for helicopter envelope expansion and performance improvement," Journal of the American Helicopter Society, Vol. 59, No. 1, 2014, pp. 1-10.

-[18] Moser, P., Barbarino, S., and Gandhi, F., "Helicopter Rotor-Blade Chord Extension Morphing Using a Centrifugally Actuated Von Mises Truss," Journal of Aircraft, Vol. 51, No. 5, 2014, pp. 1422-1431.

[19] Prabhakar, T., Gandhi, F., Steiner, J., and McLaughlin, D., “A centrifugal force actuated variable span morphing helicopter rotor," Annual Forum Proceedings - AHS International, Vol. 1, 2007, pp. 710-724.

[20] Werter, N., Sodja, J., Spirlet, G., and De Breuker, R., "Design and Experiments of a Warp Induced Camber and Twist Morphing Leading and Trailing Edge Device,” 24th AIAA/AHS Adaptive Structures Conference, 2016, pp. 1-20.

[21] Zahoor, Y., Sodja, J., De Breuker, R., and Voskuijl, M., "Development and Testing of an Active Trailing Edge Morphing Demonstrator for a Rotary Wing,” Vol. ASME 2021 Conference on Smart Materials, Adaptive Structures and Intelligent Systems, 2021.

[22] Vidyarthi, K., Zahoor, Y., Pavel, M. D., Voskuijl, M., and de Breuker, R., “Active and passive camber morphing for helicopter rotors towards performance improvements in hover and vertical flight," Vertical Flight Society's 76th Annual Forum and Technology Display, 2020.

[23] Vidyarthi, K., Beuker, M., Yin, F., Voskuijl, M., and Pavel, M., "Hoplite - a conceptual design environment for helicopters incorporating morphing rotor technology," Proceedings of the 44th European Rotorcraft Forum, edited by C. Hermans, 2018. 44th European Rotorcraft Forum, ERF 2018 ; Conference date: 18-09-2018 Through 21-09-2018.

[24] van Holten, T., and Melkert, J. A., “Helicopter Performance, Stability and Control,” Tech. Rep. November, 2002.

[25] Rauleder, J., Van Der Wall, B. G., Abdelmoula, A., Komp, D., Kumar, S., Ondra, V., Titurus, B., and Woods, B. K., “Aerodynamic performance of morphing blades and rotor systems," Annual Forum Proceedings - AHS International, Vol. 2018-May, 2018.

[26] Gandhi, F., Duling, C., and Straub, F., "On power and actuation requirement in swashplateless primary control of helicopters using trailing-edge flaps," The Aeronautical Journal (1968), Vol. 118, No. 1203, 2014, p. 503-521.

[27] Khoshlahjeh, M., Baet, E. S., Gandhi, F., and Webster, S., "Helicopter performance improvement with variable chord morphing rotors," 36th European Rotorcraft Forum, ERF 2010, Vol. 2, 2010, pp. 919-930. 
[28] Zahoor, Y., Sodja, J., De Breuker, R., and Voskuijl, M., “Actuation Device for Actuating a High-lift Device of a Rotor Blade,” , TU Delft Patent N2029203, Sep.2021.

[29] Timoshenko, S., and Krieger, S. W., Theory of Plates and Shells, McGraw Hill Book Company, 1989.

[30] Kollár, L. P., and Springer, G. S., Mechanics of Composite Structures, Cambridge University Press, 2003.

[31] Walz, C., and Chopra, I., "Design and testing of a helicopter rotor model with smart trailing edge flaps," 1994.

[32] Paredes, M., Stephan, T., and Orcière, H., "Enhanced Formulae for Determining the Axial Behavior of Cylindrical Extension Springs," Mechanics and Industry,EDPSciences, , No. 1, 2019. 